\title{
COMPACTACIÓN DEL SUELO DURANTE LA COSECHA FORESTAL. EVOLUCIÓN DE LAS PROPIEDADES FÍSICAS
}

\author{
Roberto Hernán Balbuena ${ }^{1}$, Antonino Marcelo Terminiello ${ }^{1}$, Jorge Alejandro Claverie ${ }^{1}$, \\ Juan Pablo Casado ${ }^{1}$ \& Raúl Marlats ${ }^{2}$
}

\begin{abstract}
RESUMEN
Se realizaron ensayos en campo con el objeto de evaluar los efectos y la persistencia de la compactación inducida por diferentes intensidades de tránsito durante la cosecha forestal en una población de álamos (Populus sp.). Se trabajó con un tractor de tracción simple de 73,5 kW y un acoplado, con una masa de 4,2 Mg y 2,7 Mg, respectivamente. Se establecieron 3 tratamientos de tránsito: 1,5 y 10 pasajes sobre una misma senda, contrastados con un testigo sin tránsito. Luego de cada tratamiento, y 3 años después de realizados los mismos, se efectuaron determinaciones de penetrometría, densidad aparente y humedad gravimétrica. Hubo incrementos significativos de resistencia a la penetración para los tratamientos con tránsito, alcanzando el de mayor intensidad valores entre el 76 y el $16 \%$ en los primeros $40 \mathrm{~cm}$ de profundidad. La densidad aparente tuvo una tendencia similar limitada a una menor profundidad. Luego de 3 años, los efectos persistían a nivel subsuperficial, para el índice de cono en los sectores tránsitados con respecto a los no transitados, encontrándose diferencias significativas para el tratamiento de 10 pasajes respecto al de 1 pasaje hasta $50 \mathrm{~cm}$. Aún con cargas por eje de $3 \mathrm{Mg}$, el incremento de la intensidad de tránsito induce compactación a nivel subsuperficial que persiste en el tiempo.
\end{abstract}

Palabras clave: intensidad de tránsito, índice de cono, densidad aparente

\section{SOIL COMPACTION BY FORESTRY HARVESTER OPERATION. EVOLUTION OF PHYSICAL PROPERTIES}

\begin{abstract}
The long-term effects on soil compaction by different traffic intensities during forestry harvester operation were evaluated in field experiments on heavy clay, vertic Argiudol. A conventional 2WD $73.5 \mathrm{~kW}$ engine power tractor with a trailer, $4.2 \mathrm{Mg}$ and $3.75 \mathrm{Mg}$ total load respectively, were used on a multiclonal Populus deltoides (Marsh) 3x3 plantation. There were four treatments: one pass, five pass and ten repeated passes with the tandem over the same track, and a control plot without experimental traffic. Moisture content, bulk density and penetrometer resistances were evaluated before and immediately after the traffic treatments and three years later. Effects of induced soil compaction by traffic were especially marked on topsoil, with cone index increments between 76 and $16 \%$ in the first $40 \mathrm{~cm}$ of the surface layer after treatments. Bulk density had a similar tendency but limited to a $15 \mathrm{~cm}$ depth. Three years later, only subsoil cone index in the track line, below $20 \mathrm{~cm}$ depth, was significantly higher than the interline track soil cone index. The 10 passes treatment had significant differences compared to the 1 pass and no traffic control plot treatments from the surface layer to $50 \mathrm{~cm}$ depth, without differences with the 5 passes treatment in most of the studied soil layers. Subsoil compaction may be induced by repeated traffic with low axle load and their effects persist for a very long time.
\end{abstract}

Key words: traffic intensity, cone index, bulk density

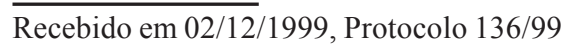

${ }^{1}$ Docentes-investigadores de la Facultad de Cs Agrarias y Forestales de la Universidad Nacional de La Plata. Avda 60 y 119 (1900) La Plata, Argentina. Fax 54-221-4252346. E-mail balbuena@isis.unlp.edu.ar

${ }^{2}$ Docentes-investigadores de la Facultad de Cs Agrarias y Forestales de la Universidad Nacional de La Plata. Avda 60 y 119 (1900) La Plata, Argentina. Fax 54-221-4252346. CICPBA, Av. da Antártida Argentina y 526 (1900) La Plata. 


\section{INTRODUCCIÓN}

El constante y significativo aumento del peso de los vehículos agrícolas y forestales registrado en las últimas décadas, preocupa por las consecuencias a largo plazo sobre la productividad de los suelos transitados, con implicancias en la conservación de los recursos. Debido a esto, el potencial de daños a la productividad del sitio es grande en los bosques ya que ésta operación se repite en forma recurrente (Matthes et al., 1988). El acortamiento de los turnos de cosecha y la regularidad de abastecimiento que el mercado exige, induce a los responsables del aprovechamiento a incursionar dentro de las plantaciones cuando las condiciones edáficas no son apropiadas, por lo que se acentúan los daños que sufre el suelo. Entre los mismos se cita a la compactación, definida por Marshall \& Holmes (1988), como el proceso por el cual un suelo no saturado es comprimido por una carga aplicada durante un lapso breve sin expulsión de agua. Por lo tanto, la resistencia del suelo no depende solamente de las propiedades de las partículas y la estructura del suelo, sino también de los contenidos de agua y aire, y sus posibilidades de fluir por la matriz del suelo (Beekman, 1987). El tráfico del rodado en el terreno ha sido reconocido como la mayor fuente de fuerzas causantes de la compactación (Schafer et al., 1989). Los perjuicios que causa la compactación son notados principalmente en los sectores donde circulan los vehículos. Como consecuencia de la misma, el escurrimiento superficial se incrementa y el crecimiento de los árboles es perjudicado (Rab, 1992).

Las operaciones de cosecha forestal poseen como característica distintiva con respecto a otras, el pasaje repetido de los vehículos o conjuntos sobre un mismo sector. Dado que existen diferencias en relación a la posición del ramal en el área trabajada, se verifican diferentes intensidades de tránsito de acuerdo a la proximidad a los patios (Firth \& Murphy, 1989). Mac Donagh (1994) estimó un rango de intensidades de tránsito para distintos sectores del monte durante la cosecha forestal de 168 a $196 \mathrm{Mg} \mathrm{km} \mathrm{ha}^{-1}$, en promedio de toda la plantación. Estos valores pueden ser mayores, en la medida que se consideren solamente los lugares cercanos a los patios

El tránsito repetido en una misma senda provoca daños en la estructura del suelo que causa reducciones en el rendimiento tanto en el primer año de producido el mismo, como así también decrecimiento en años posteriores (Håkansson \& Reeder, 1994). Cuando el tránsito se realiza en condiciones de suelo húmedo, el resultado negativo se verá maximizado y la profundidad de la zona afectada será mayor (Voorhees et al., 1989). Si bien la primera pasada suele considerarse como la que induce los mayores cambios, esto se restringe a condiciones de suelos sueltos, recién labrados (Taylor et al., 1982). En cambio, cuando el suelo se encuentra consolidado el grado de compactación puede ser similar para las distintas pasadas (Sánchez Girón Renedo, 1996).

Según Kobayashi (1994), cuando el peso de los troncos y la separación entre árboles no permite la utilización del sistema de acarreo por cable aéreo, el tránsito de tractores y acoplados causa los cambios más severos en la estructura del monte y del suelo. Greacen \& Sands (1980) evaluaron las causas y efectos de la compactación forestal, determinando que el arrastre de troncos afectó el suelo en una instancia hasta una profundidad de $30 \mathrm{~cm}$, mientras que en la vía de saca, el suelo resultó compactado hasta $50 \mathrm{~cm}$ de profundidad.

De acuerdo con los conocimientos actuales los procesos de compactación del suelo son sumamente complejos. Para un suelo dado, la distribución de la compactación en el perfil es función de la carga aplicada, la presión ejercida por el rodado, el estado de humedad y la intensidad de tránsito recibida (Raper \& Erbach, 1990). Raper et al. (1994) analizaron diversas investigaciones y establecieron que la tensión de mayor impacto en el daño producido dentro del perfil sometido a compactación inducida por tránsito, es la que ocurre en sentido vertical.

Smith \& Dickson (1990) analizaron diversos trabajos de investigación y demostraron que la presión de contacto influye sobre la compactación superficial. Sin embargo, la compactación del subsuelo, por debajo de $40 \mathrm{~cm}$ de profundidad, está directamente influenciada por el peso de los vehículos, en forma independiente de la presión de contacto rueda suelo. Håkansson et al. (1988) concluyeron que el riesgo de la compactación del subsuelo, es principalmente determinado por el peso de la rueda, aún cuando la presión de contacto fuese baja. Citaron además, ejemplos de compactaciones por debajo de los $40 \mathrm{~cm}$, producidas por cargas sobre el eje mayores a $6 \mathrm{Mg}$. Además, establecieron que compactaciones por debajo de $50 \mathrm{~cm}$ de profundidad, producidas por cargas mayores a $10 \mathrm{Mg} / \mathrm{eje}$, pueden perdurar por 7 años o más. Estos datos se corresponden con la recomendación de Håkansson \& Danfors (1981) de limitar el peso de un eje simple a no más de $6 \mathrm{Mg}$, a los efectos de prevenir la compactación subsuperficial. En contraste con estos resultados, Aura (1983) determinó que aún con cargas sobre el eje de $3 \mathrm{Mg}$ compactaron un suelo arcilloso por debajo de los $20 \mathrm{~cm}$ de capa arable, si las condiciones eran desfavorables.

Chi et al. (1992) evaluaron diferentes medidas de neumáticos en un conjunto tractor acoplado. Encontraron que el menor peso del eje causó la menor compactación, independientemente que no existiesen variaciones en la presión de contacto rueda suelo. Por otra parte, la compactación del subsuelo aumentó con cada pasaje del conjunto tractor acoplado. Gameda et al. (1987) determinaron que el pasaje repetido año tras año sobre la misma senda incrementa el grado de compactación y la profundidad de la capa compactada. Jorajuría et al. (1997) aplicaron diferentes intensidades de tránsito sobre un suelo con pastizales naturales concluyendo que la profundidad a la cual la variable peso manifiesta la mayor compactación guarda una relación inversa con el número de pasadas del tractor. Además, la misma compactación en las capas más profundas pudo alcanzarse con varios pasajes de un tractor liviano o con pocas pasadas de uno pesado. Meek (1996) estableció que en suelos arcillosos, serían mayores los riesgos de compactar el subsuelo y mayor sería también la persistencia del daño. En estos suelos, con porcentajes de arcilla entre el 6 y el $85 \%$, la compactación en el subsuelo inducida por el tráfico con altas cargas por eje era medible entre 3 y 11 años después del tránsito (Gameda et al.,1987; Logdson et al., 1992). El objetivo de este trabajo fue evaluar los efectos directos y la persistencia en el tiempo de la compactación inducida por diferentes intensidades de tránsito durante la cosecha forestal. 


\section{MATERIAL Y MÉTODOS}

El ensayo fue realizado en la Estación Experimental Julio Hirschhornn, perteneciente a la Facultad de Ciencias Agrarias y Forestales de la Universidad Nacional de La Plata, situada en Los Hornos, Provincia de Buenos Aires a los $34^{\circ} 55^{\prime}$ LS y $57^{\circ} 57^{\prime} \mathrm{L} \mathrm{O}$, con una altitud de $15 \mathrm{~m}$ sobre el nivel del mar. El suelo fue clasificado como Argiudol vértico arcilloso (Natural Resources Conservation Service, 1998), fino montmorillonítico, superficialmente franco limoso, rico en carbono orgánico, ligeramente ácido. Estructura granular, media moderada, consistencia ligeramente dura, friable, ligeramente plástica y ligeramente adhesiva.

El dosel arbóreo estaba compuesto por una plantación multiclonal de cuatro clones de Populus deltoides (Marsh), constituída por los siguientes clones: «Stoneville 66», «Catfish $2 »$, «Harvard» (I-63/51) y «Onda» (I-72/51), con una población inicial de 1111 árboles por hectárea, a un distanciamiento de $3 \times 3 \mathrm{~m}$. El corte se realizó a los 9 años, con un diámetro promedio de $18,9 \mathrm{~cm}$ y una altura media de $17,1 \mathrm{~m}$.

Se utilizó un tractor de diseño convencional (2WD), de $73,5 \mathrm{~kW}$ de potencia, con una trocha de $2,1 \mathrm{~m}$, a una velocidad de $1,5 \mathrm{~m} \mathrm{~s}^{-1}$ formando un conjunto de tracción libre con un acoplado de dos ejes, cuyas características figuran en la Tabla 1.

Durante los tratamientos, el suelo se encontraba consolidado, debido a que la última labranza sobre el mismo se realizó 2 años antes con una rastra de discos a una profundidad de $12 \mathrm{~cm}$. El suelo al momento de la realización de los ensayos se encontraba cubierto de manera uniforme por una vegetación consistente en trébol blanco (Trifolium repens) y ryegrass anual (Lolium multiflorum). El estado de humedad en el perfil estudiado resultó en promedio del $29 \%$, menor en el estrato superficial y superior a las mayores profundidades a la humedad de mayor compactabilidad del suelo, de acuerdo al Test de Proctor (Terzaghi \& Peck, 1948), según se detalla en la Tabla 2.

El diseño experimental estuvo conformado por 4 tratamientos, los cuales se visualizan en la Tabla 3. Las diferentes intensidades de tránsito están representadas por el número de pasajes del conjunto tractor acoplado, que se corresponden con diferentes vías de extracción de madera del monte. Dentro de cada parcela se efectuaron 5 repeticiones para cada tratamiento en lugares totalmente aleatorizados. Considerando $3 \mathrm{~m}$ entre hileras, cada tratamiento correspondió a intensidades de tránsito de 23,2 para un pasaje, 116 para 5 pasajes y 232 $\mathrm{Mg} \mathrm{km} \mathrm{ha-1}$ para 10 pasajes sobre el suelo.

Tabla 3. Intensidades de tránsito consideradas en el diseño experimental

\begin{tabular}{ccc}
\hline Tratamiento & $\begin{array}{c}\text { Número de } \\
\text { Pasajes } \\
\text { del Equipo: }\end{array}$ & $\begin{array}{c}\text { Intensidad } \\
\text { de Tránsito } \\
\mathrm{Mg} \mathrm{km} \mathrm{ha}^{-1}\end{array}$ \\
\hline 0P & Testigo sin tránsito & 0,0 \\
1P & Un pasaje & 23,2 \\
5P & 5 pasajes en la misma senda & 116,0 \\
10P & 10 pasajes en la misma senda & 232,0 \\
\hline
\end{tabular}

Los parámetros evaluados fueron: a) humedad del suelo por atenuación de protones y densidad aparente en seco por gammametría con una sonda troxler 3440 a profundidades de: $0-5,1 \mathrm{~cm}, 0-10,2 \mathrm{~cm}, 0-15,2 \mathrm{~cm}, 0-20,3 \mathrm{~cm}, 0-25,4 \mathrm{~cm}$ y $0-30,5 \mathrm{~cm}$, con cinco repeticiones por parcela, al año de efectuarse los tratamientos y a los 3 años de producido los mismos; b) resistencia a la penetración con un penetrómetro con unidad de almacenamiento de datos (ASAE,1992), correspondiendo cada dato de penetrometría al promedio de 10 determinaciones. En el primer momento de medición se tomaron determinaciones cada $7,6 \mathrm{~cm}$ hasta una profundidad de $38,1 \mathrm{~cm}$, mientras que en el último ciclo evaluado se efectuaron las determinaciones cada $2,54 \mathrm{~cm}$ hasta $61,0 \mathrm{~cm}$ de profundidad con un penetrómetro con unidad de registro Rimik CP 20 construído también bajo norma S.313 (ASAE, 1992); c) Humedad gravimétrica en intérvalos de $10,0 \mathrm{~cm}$ hasta la profundidad de medición del penetrómetro en cada ciclo de medición; d) ensayo de Compactación Proctor ASTM-D-698 T.

El análisis de los datos se realizó como multifactorial y la significancia de las diferencias evaluadas por el test de Tuckey

\section{RESULTADOS Y DISCUSIÓN}

Los incrementos de la resistencia a la penetración después de cada tratamiento con relación al testigo se observan en la

Tabla 1. Características de los vehículos empleados para la realización de los tratamientos con tránsito

\begin{tabular}{lccccc}
\hline \multirow{2}{*}{ Características } & \multicolumn{2}{c}{ Tractor } & & \multicolumn{2}{c}{ Acoplado } \\
\cline { 2 - 3 } \cline { 5 - 6 } & Tren Trasero & Tren Delantero & & Eje Trasero & Eje Delantero \\
\hline Peso $(\mathrm{Mg})$ & 2,8 & 1,4 & 1,375 & \\
Volumen de carga total $\left(\mathrm{m}^{3}\right)$ & & & 7,375 \\
Rodado: & $18,4-34$ & $7,50-16$ & & $7,50-16$ & $7,50-16$ \\
Presión de inflado $(\mathrm{kPa})$ & 128 & 210 & 280 & 280 \\
Área de contacto rueda/suelo $\left(\mathrm{m}^{2}\right)$ & 0.182 & 0.0646 & & 0.0646 & 0.0646 \\
Presión de contacto $(\mathrm{kPa})$ & 73 & 107 & & 104.3 & 104.3 \\
Ancho total & 2,10 & 2,10 & & 2,10 & 2,10 \\
\hline
\end{tabular}

Tabla 2. Humedad al momento de realización de los tratamientos y ensayo de Compactación Proctor del suelo en estudio

\begin{tabular}{ccccc}
\hline $\begin{array}{c}\text { Profundidad } \\
(\mathrm{cm})\end{array}$ & $\begin{array}{c}\text { Materia Orgánica } \\
\%\end{array}$ & $\begin{array}{c}\text { Humedad }^{1} \\
\%\end{array}$ & $\begin{array}{c}\text { Humedad }^{2} \\
\%\end{array}$ & $\begin{array}{c}\text { Máxima Densidad }^{2} \\
\text { Aparente, Mg m }^{-3}\end{array}$ \\
\hline $0-15$ & 3,3 & 26 & 29 & 1,54 \\
$15-30$ & 1,7 & 28 & 22 & 1,78 \\
$30-60$ & 1,2 & 33 & 18 & 1,80 \\
\hline
\end{tabular}

Al momento de efectuar el tránsito (base peso seco)

Humedad y densidad aparente correspondientes al Test de Proctor (base peso seco) 
Figura 1. En el rango de profundidades evaluadas en esta instancia de medición, los incrementos en resistencia a la penetración fueron más importantes a nivel superficial. Estos resultados concuerdan con los obtenidos por Smith \& Dickson (1990) y Håkansson \& Danfors (1981). De acuerdo con las cargas por eje del tractor y del acoplado utilizados, inferiores al valor de $6 \mathrm{Mg}$ indicado por Håkansson et al. (1988) que produciría compactación por debajo de los $40,0 \mathrm{~cm}$ de profundidad. Los mayores efectos estuvieron por encima de esta profundidad y es posible relacionarlos con las presiones de contacto rueda suelo, superiores a $100 \mathrm{kPa}$ en 3 de los 4 ejes del conjunto tractor-acoplado.

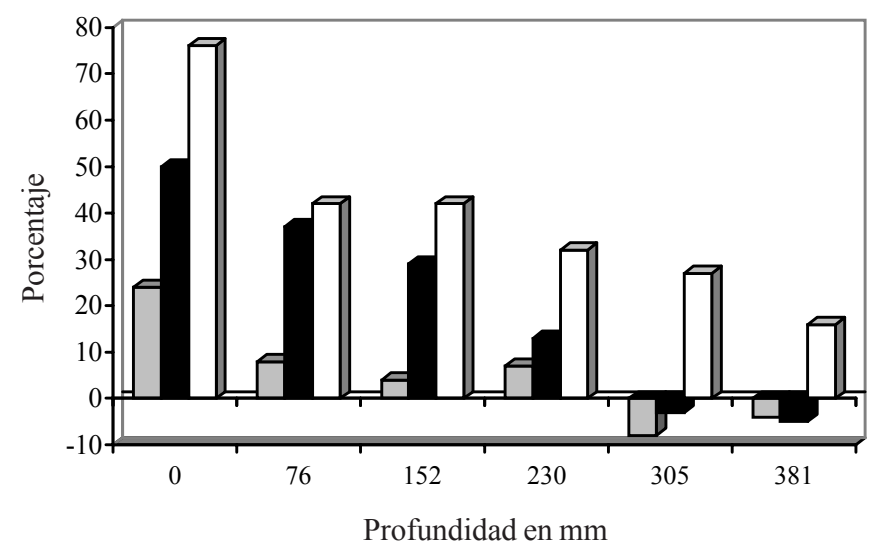

\section{口1P $\square 5 P \quad \square 10 P$}

Figura 1. Incrementos del índice de cono con respecto al testigo en porcentaje inmediatamente posterior a la realización de los tratamientos

Con respecto al número de pasadas sobre el terreno, es posible afirmar que el número de pasadas sobre el terreno incrementó la profundidad a la cual se encuentran diferencias con el testigo. Esto resulta coincidente con los resultados de Chi et al. (1992) y Jorajuría et al. (1997), en relación con el incremento de la compactación subsuperficial con el número de pasajes, en forma independiente de la presión superficial. Sin embargo, esto resultó claro solamente para el tratamiento de 10 pasajes del conjunto, puesto que los valores de resistencia a la penetración a $30,0 \mathrm{~cm}$ de profundidad y a $40,0 \mathrm{~cm}$ de profundidad para 1 y 5 pasajes mostraron un pequeño decrecimiento con respecto al testigo. Esto podría deberse en parte, al contenido de humedad del primer rango de profundidad estudiado, el cual resultó muy cercano a la humedad del suelo en la que se alcanza el mayor grado de compactación. Por otra parte, dada la capacidad portante del suelo al momento de realizarse los ensayos y a las características texturales del mismo, el mayor efecto de compactación no ocurrió durante el primer pasaje del conjunto como determinó Taylor et al.(1982), para suelos sueltos, recién labrados, salvo para la resistencia a la penetración a nivel de superficie. Entre 7,6 y 15,2 cm, el grado de compactación resultó aproximadamente similar en promedio para cada pasaje, tal como fue sugerido por Sánchez Girón Renedo (1996).

Los valores de densidad aparente, determinados luego de realizados los tratamientos de tránsito se visualizan en la Tabla 4, y tienen una tendencia similar a la de la resistencia a la penetración para los primeros $15,0 \mathrm{~cm}$, pero no así en las mayores profundidades.

Luego de 3 años de haberse efectuado los tratamientos, no fue posible ubicar visualmente las huellas producidas por el tránsito vehicular. Por lo tanto, se debió determinar los sectores transitados y no transitados a partir de los sitios identificados originalmente para delimitación de las parcelas y las medidas de los vehículos utilizados.

En relación con la humedad del suelo de los distintos tratamientos con tránsito, se determinó significancia para la interacción posición, dentro y fuera de la huella, y profundidad, por lo que se procedió a analizar cada posición en forma independiente. Las diferencias de tendencias entre las posiciones dentro y fuera de los sectores transitados, se encuentran limitadas a los primeros $20,0 \mathrm{~cm}$, donde dentro de la huella se registran mayores contenidos de humedad que entre las huellas. Para la posición delimitada entre las huellas del conjunto tractor acoplado, no se encontraron diferencias significativas en la totalidad del perfil. La humedad fue muy uniforme entre los tratamientos registrándose solamente una diferencia del $5 \%$ para el tratamiento de 10 pasadas con respecto a los restantes en el estrato de 0 a $10,0 \mathrm{~cm}$, correspondiendo la mayor humedad a la mayor intensidad de tránsito. En cuanto a las mediciones efectuadas en la huella de los vehículos, el gradiente de humedad fue similar para los 3 estratos superiores, en forma inversa a lo registrado para la posición entre las huellas. En promedio, el tratamiento de 10 pasadas tuvo en los primeros $20,0 \mathrm{~cm}$ aproximadamente un $4 \%$ menos humedad que los otros subtratamientos. Estas diferencias se reducen en el tercer estrato y son inferiores al $1 \%$ a partir de $10 \mathrm{~s} 30,0 \mathrm{~cm}$. Los tratamientos de 1 y 5 pasadas fueron en todo el perfil muy similares.

En cuanto a los valores de resistencia a la penetración expresados como índice de cono, no se encontraron interacciones entre los tratamientos de tránsito, posiciones y profundidades. El análisis de los resultados indica diferencias significativas para las posiciones entre las huellas y en las huellas del tractor, correspondiendo a esta última los mayores valores medios (Tabla 5).

Por debajo de los 2 primeros estratos, surge claramente la incidencia del efecto del tránsito sobre el índice de cono, con incrementos variables del $11,12,11,45,18,83$ y $12,35 \%$ para los 4 estratos delimitados entre 20,0 y $60,0 \mathrm{~cm}$ respectivamente y

Tabla 4. Valores de densidad aparente en $\mathrm{Mg} \mathrm{m}^{-3}$, medidos en 6 rangos de profundidad después del tránsito

\begin{tabular}{clccccc}
\hline Tratamientos & $0-5 \mathrm{~cm}$ & $0-10,2 \mathrm{~cm}$ & $0-15,2 \mathrm{~cm}$ & $0-20,3 \mathrm{~cm}$ & $0-25,4 \mathrm{~cm}$ & $0-30,5 \mathrm{~cm}$ \\
\hline Testigo & $1.125 \mathrm{a}$ & $1.145 \mathrm{a}$ & $1.151^{\mathrm{a}}$ & $1.181 \mathrm{a}$ & $1.202 \mathrm{a}$ & $1.246 \mathrm{a}$ \\
$1 \mathrm{P}$ & $1.152 \mathrm{ab}$ & $1.166 \mathrm{ab}$ & $1.171 \mathrm{ab}$ & $1.195 \mathrm{a}$ & $1.214 \mathrm{a}$ & $1.268 \mathrm{~b}$ \\
$5 \mathrm{P}$ & $1.161 \mathrm{ab}$ & $1.173 \mathrm{ab}$ & $1.175 \mathrm{ab}$ & $1.198 \mathrm{a}$ & $1.210 \mathrm{a}$ & $1.240 \mathrm{a}$ \\
$10 \mathrm{P}$ & $1.188 \mathrm{~b}$ & $1.186 \mathrm{~b}$ & $1.192 \mathrm{~b}$ & $1.206 \mathrm{a}$ & $1.210 \mathrm{a}$ & $1.250 \mathrm{ab}$ \\
\hline \multicolumn{2}{l}{ Valores medios seguidos de diferente letra indican diferencias estadísticamente significativas entre tratamientos y de cada tratamiento con respecto al testigo a la misma profundidad de acuerdo al test }
\end{tabular}


Tabla 5. Valores de índice de cono cada 10,0 cm de profundidad para los sectores del suelo entre las huellas y en la huella de los tratamientos con tránsito

\begin{tabular}{lcccccc}
\hline Posición & $0-10,0$ & $10,1-20,0$ & $20,1-30,0$ & $30,1-40,0$ & $40,1-50,0$ & $50,1-60,0$ \\
\hline Medio & $769,58 \mathrm{a}$ & $885,05 \mathrm{a}$ & $881,18 \mathrm{a}$ & $864,15 \mathrm{a}$ & $1161,48 \mathrm{a}$ & $1628,70 \mathrm{a}$ \\
Huella & $815,68 \mathrm{a}$ & $957,09 \mathrm{a}$ & $979,08 \mathrm{~b}$ & $963,15 \mathrm{~b}$ & $1380,19 \mathrm{~b}$ & $1829,94 \mathrm{~b}$ \\
\hline
\end{tabular}

Valores medios seguidos de letras diferentes en cada columna indican diferencias significativas de acuerdo al test de Tukey $(\mathrm{P} \leq 0,05)$

concuerdan con lo citado por Gameda et al. (1987) y Logdson et al. (1992) en cuanto a la persistencia del efecto del tránsito sobre suelos arcillosos. Los resultados confirman, a su vez, lo expresado por Meek (1996) sobre los riesgos de compactación y persistencia del efecto para este tipo de suelos. Las diferencias de índice de cono entre los sectores bajo las ruedas y entre las ruedas del tractor, detectables a nivel subsuperficial tres años después de efectuados los tratamientos no habían podido ser determinadas anteriormente debido a las limitaciones de profundización del instrumental de medición con que se contaba originalmente. El tránsito de tractores y acoplados, en concordancia con lo expresado por Kobayashi (1994), ha sido el causante de los cambios acaecidos en la estructura del suelo. Los resultados también coinciden con los encontrados por Greacen \& Sands (1980), en relación con la profundidad a la que los efectos pueden ser detectados, llegando los mismos en este caso hasta los $60,0 \mathrm{~cm}$ de profundidad.

$\mathrm{La}$ ausencia de significancia a nivel superficial (entre $0 \mathrm{y}$ $20,0 \mathrm{~cm}$ de profundidad), podría asociarse a la mayor humedad gravimétrica que registró la posición dentro de la huella. Esto resulta importante si se tiene en cuenta que en el estrato de 0 a $10,0 \mathrm{~cm}$, la humedad para el subtratamiento dentro de la huella fue del orden del $8 \%$ mayor, y entre el 3 y el $5 \%$ aproximadamente superior para igual posición entre los 10,0 y $20,0 \mathrm{~cm}$., para los tratamientos de 1 y 5 pasadas. Esto indicaría que, de haber resultado similares los valores de humedad gravimétrica, se podría haber alcanzado también diferencias significativamente mayores para el índice de cono determinado en el sector transitado por las ruedas con respecto al que resulta intermedio entre el pasaje de las mismas.

Para el tratamiento con mayor intensidad de tránsito, no existieron diferencias de humedad en todo el perfil. Por lo tanto, los incrementos en la resistencia a la penetración registrados para este tratamiento sobre la huella con respecto al sector entre las huellas en la totalidad de las profundidades, debe asignarse exclusivamente a la mayor incidencia de la compactación inducida en el suelo en sentido vertical con respecto a la radial.

En cuanto al análisis entre los tratamientos de tránsito para las distintas profundidades sobre el sector transitado por las ruedas del conjunto tractor-acoplado (Tabla 6), se encontraron diferencias significativas entre el tratamiento con 1 pasaje sobre el suelo y el de 10 pasajes para la totalidad de las profundidades hasta $\operatorname{los} 50,0 \mathrm{~cm}$.
Por lo expuesto, tal como indican Håkansson \& Reeder (1994), el tránsito repetido en la misma senda es el determinante de los daños en la estructura del suelo en profundidad en los aspectos físico-mecánicos. La persistencia del mismo a nivel subsuperficial, tal como se desprende de los resultados alcanzados a 3 años de haberse realizado los trabajos experimentales, implica riesgos en el desarrollo posterior de plantaciones forestales, en la capacidad de exploración radicular, si es que no se efectúan trabajos de descompactación profunda del suelo. Si bien tal como fue indicado por Voorhees (1989) la compactación se verá maximizada en condiciones de suelo húmedo, los efectos sobre el suelo se manifestaron en este caso a nivel subsuperficial, pese a que el estado de humedad al plantearse el ensayo para los estratos del terreno por debajo de los 30,0 cm se encontraban marcadamente alejados a los determinados por el test de Proctor (Terzaghi \& Peck 1948), como aquellos en los cuales se alcanza la máxima compactación del sustrato. La significancia de las diferencias entre los sectores del suelo entre las huellas y dentro de las huellas de los vehículos confirman lo expuesto por Raper \& Erbach (1990), en cuanto a la incidencia de la intensidad de tránsito sobre la compactación del suelo y Raper et al. (1994) en relación con que la tensión en sentido vertical, es la de mayor impacto sobre el suelo.

En función de los resultados de la Tabla 6 merece destacarse que el número de pasadas sobre el terreno, correspondientes a la mayor intensidad de tránsito, tal como fuera afirmado por Jorajuría et al.(1997), produjo modificaciones en el estado físico mecánico del suelo a nivel subsuperficial, independientemente de que la masa sobre cada eje tanto del tractor como del acoplado fuesen inferiores a $6 \mathrm{Mg}$, indicadas por Smith \& Dickson (1990), Håkansson et al. (1988) y Håkansson \& Danfors (1981). Esto afirma a su vez lo puntualizado por Aura (1983), en cuanto a las posibilidades de compactar el suelo por debajo de $20,0 \mathrm{~cm}$ de profundidad, aún con masas de $3 \mathrm{Mg}$. Por otra parte, en la primera instancia de medición no fue posible detectar, tal como concluyeran Jorajuría et al.(1997), que el mayor aumento relativo de resistencia a la penetración tuviese una relación inversa con el número de pasadas. En cambio, los resultados concuerdan con lo expresado por Gameda et al. (1987) en lo referente al incremento del grado de compactación y la mayor profundidad de la capa compactada, en la medida que se incrementó el número de pasadas por la misma senda. En cuanto a la densidad aparente, no se encontraron diferencias

Tabla 6. Índice de cono cada $10,0 \mathrm{~cm}$ de los tratamientos con tránsito en la zona transitada por los rodados del conjunto tractor acoplado

\begin{tabular}{clrrlll}
\hline Tratamiento & $0-10,0$ & $10,1-20,0$ & $20,1-30,0$ & $30,1-40,0$ & $40,1-50,0$ & $50,1-60,0$ \\
\hline $1 \mathrm{P}$ & $726,12 \mathrm{a}$ & $824,79 \mathrm{a}$ & $774,79 \mathrm{a}$ & $836,9 \mathrm{a}$ & $1136,10 \mathrm{a}$ & $1832,14 \mathrm{~b}$ \\
$5 \mathrm{P}$ & $764,47 \mathrm{ab}$ & $850,87 \mathrm{a}$ & $956,60 \mathrm{~b}$ & $925,66 \mathrm{ab}$ & $1318,62 \mathrm{ab}$ & $1532,93 \mathrm{a}$ \\
$10 \mathrm{P}$ & $887,29 \mathrm{~b}$ & $1087,56 \mathrm{~b}$ & $1059,00 \mathrm{~b}$ & $978,35 \mathrm{~b}$ & $1357,79 \mathrm{~b}$ & $1822,89 \mathrm{~b}$ \\
\hline
\end{tabular}


significativas entre tratamientos en ningún estrato entre $0 \mathrm{y}$ $30,0 \mathrm{~cm}$ de profundidad, confirmando lo expresado por Alakuku (1997), sobre la escasa sensibilidad de este parámetro para la detección de compactación inducida por tránsito.

En cuanto a los estratos superiores, hasta $20,0 \mathrm{~cm}$ de profundidad, más cercanos a la humedad de mayor compactabilidad del suelo al momento de realizarse los tratamientos de tránsito, aparecen como los menos afectados luego de pasados 3 años, contrariamente a lo encontrado en la primera instancia de medición, con diferencias solamente para el tratamiento de mayor intensidad de tránsito. Los tratamientos de 1 y 5 pasajes sobre el terreno, no alcanzaron diferencias significativas con el testigo sin tránsito, lo cual podría atribuirse en este caso, a los procesos de humectación y desecación, como así también a la mayor bioactividad del suelo a nivel superficial, en concordancia con lo expresado por Alakuku (1997).

En función de lo expuesto, el tránsito repetido en una misma senda, producto del proceso de cosecha y extracción de la madera, podría causar efectos sobre plantaciones forestales posteriores a la misma en un determinado sitio, tanto por sus efectos sobre la compactación superficial del suelo en el corto plazo, como por su incidencia en la compactación subsuperficial, aún cuando las operaciones se realicen con bajas cargas sobre el eje de los vehículos utilizados.

\section{CONCLUSIONES}

1. El pasaje repetido de vehículos con baja carga por eje, incrementa el índice de cono subsuperficialmente, cuando la condición del suelo a nivel superficial favorece los procesos de compactación.

2. Cuando se incrementa la intensidad de tránsito por aumentos del número de pasajes, aumenta el grado de compactación y la profundidad de la capa compactada en forma independiente de la carga sobre el rodado.

\section{REFERENCIAS BIBLIOGRÁFICAS}

ALAKUKU, L. Long-term soil compaction due to high axle load traffic. Vammalan Kirjapaino Oy: Agricultural Research Centre of Finland Institute of Crop and Soil Science, 1997. 461p. Tesis Doctoral

ASAE. Standards of the ASAE 1992. S.313.2 Soil cone penetrometer. St Joseph, Michigan: American Society of Agricultural Engineering, 1992.

AURA, E. Soil compaction by the tractor and its effects on soil porosity. Journal of Scientific Agricultural Society of Finland, Helsinki, v. 55, p.91-107, 1983.

BEEKMAN, F. Soil strength and forest operations. Department of Forest Technique and Forest Products. Wageningen, The Netherlands: Agricultural University, 1987. 168p.

CHI, L.S.; TESSIER, S.; LAGË, C. Finite element modelling of soil compaction by liquid manure spreaders. Transactions of the ASAE, St. Joseph, MI, v.36, n.3, p.637-644, 1992.

FIRTH, J.; MURPHY, G. Skidtrails and their effect on the growth and management of young Pinus radiata. New Zeland Journal Forestry Science, Rotorua, v.19, v.1, p.22-28, 1989.
GAMEDA, S., RAGHAVAN, G.S.V., MCKYES, E.; THERIAULT, R. Subsoil compaction in a clay soil. I. Cumulative effects. Soil and Tillage Research, Amsterdam, v.10, p.113-122, 1987. GRACEN, E.L.; SANDS, R. Compaction of forest soils. A review. Australian Journal of Soil Research, Sidney, v.18, p.163-189, 1980.

HÅKANSSON, I.; DANFORS, B. Effects of heavy traffic on soil conditions and crop growth. In: Proc. 7th International Conference of ISTVS, Canada. Hanover, NH, USA: International Society for Terrain-Vehicle Systems (ISTVS), 1981. p. 239-253.

HÅKANSSON, I.; REEDER, R.C. Subsoil compaction by vehicles with high axle load-extent, persistence and crop response. Soil \& Tillage Research, Amsterdam, v.29, p.277-304, 1994.

HÅKANSSON, I.; VOORHEES, W.B.; RILEY, H. Vehicle and wheel factors influencing soil compaction and crop response in different traffic regimes. Soil and Tillage Research, Amsterdam, v.11, p.239-282, 1988.

JORAJURIA, D.; DRAGHI, L.; ARAGON, A. The effect of vehicle weight on the distribution of compaction with depth and the yield of Lolium/Trifolium grassly. Soil and Tillage Research, Amsterdam, v.41, p.1-12, 1997.

KOBAYASHI, S. Effects of harvesting impacts and rehabilitation of tropical rain forest. Journal of Plant Research, Tokyo, v.107, p.99-106, 1994.

LOGSDON, S.; ALLMARAS, R.R.; NELSON, W.W.; VOORHEES, W.B. Persistence of subsoil compaction from heavy axle loads. Soil and Tillage Research, Amsterdam, v.23, p.93-110, 1992.

Mac DONAGH, P. Economic and technical evaluation of Pinus $s p$ harvesting with crawled tractors in south Brazil. Federal University of Parana, Brazil. 1994. 180p. Master Dissertation MARSHALL, T.J.; HOLMES, J.W. Soil physics. $2^{\text {nd }}$ ed. Reading: Cambridge University Press, 1994. 374p.

MATTHES, R.; WATSON, W.; SAVELLE, I.; SIROIS, D. Effect of load and speed on fuel consumption of a rubber tired skidder. Transaction of ASAE, St. Joseph, MI, v. 31, n. 1, p.37-39, 1988.

MEEK, F.E. Effects of skidder traffic on two types of forest soils. Forest Engineering Research Institute of Canada. 12p. 1996. Technical Report TR117

NATURAL RESOURCES CONSERVATION SERVICE. Keys to soil taxonomy. 8th edition. Washington D.C.: Soil Survey Staff, USDA, 1994. 328p.

RAB, M.A. Impact of timber harvesting and compaction with reference to residual log harvesting in East Gippsly, Victoria- A review. Native Forest Research. Department of Conservation and Environment, Victoria, Australia, 18p, 1992. VSP Technical Report $N^{\circ} 13$

RAPER, R.; ERBACH, D. Prediction of soil stresses using the finite element method. Transactions of the ASAE, St. Joseph, MI, v.33, n.3, p.725-730, 1990.

RAPER, R.L.; JOHNSON, C.E.; BAILEY, A.C. Coupling normal and shearing stresses to use in finite element analysis of soil compaction. Transactions of the ASAE, St. Joseph, MI, p.37, v.5, p.1417-1422, 1994. 
SÁNCHEZ GIRÓN RENEDO, V. Compactación del suelo agrícola. En: Dinámica y mecánica de suelos. Madrid, Ediciones Agrotécnicas S.L., 1996. p.269-303

SCHAFER, R.L.; BAILEY, A.C.; JOHNSON, C.E.; RAPER, R.L. A rationale for modeling soil compaction behavior: An engineering mechanics approach. ASAE Paper No 89-1097, St. Joseph, MI, 1989.

SMITH, D.L.O.; DICKSON J.W. The contribution of vehicle weight and ground pressure to soil compaction. Journal of Agricultural Engineering Research, London, v.46, p.13-29, 1990.
TAYLOR, J.; BURT, E.; BAILEY, N. Multipass behavior of a neumatic tyre in tilled soils. ASAE Paper No79-1549, St. Joseph, MI, 1982.

TERZAGHI, K.; PECK, R.B. 1948. Soil mechanics in engeneering practice. 1.ed. New York: John Wiley and Sons, 1948. 566p.

VOORHEES, W.B.; JOHNSON, J.F.; RYALL, G.W.; NELSON, W.W. Corn growth and yield as affected by surface and subsoil compaction. Agronomy Journal, Madison, v.81, p.294-303, 1989. 